\title{
Percutaneous femoral arterial and venous catheterisation during neonatal intensive care
}

\author{
S P Wardle, A W R Kelsall, C W Yoxall, N V Subhedar
}

\begin{abstract}
Background-Femoral vessel catheterisation is generally avoided in the neonatal period because of technical difficulties and the fear of complications.

Aim-To review the use of femoral arterial and venous catheters inserted percutaneously on the neonatal intensive care unit. Methods-Infants admitted to one of two regional neonatal intensive care units who underwent femoral vessel catheterisation were identified. Information collected included basic details, indication for insertion of catheter, type of catheter and insertion technique, duration of use, and any catheter related complications.

Results-Sixty five femoral catheters were inserted into 53 infants. The median gestational age was 29 weeks (range 2340). Twenty three femoral arterial catheters (FACs) were inserted into 21 infants and remained in situ for a median of three days (range one to eight). Twelve (52\%) FACs remained in place until no longer required, and four (17\%) infants developed transient ischaemia of the distal limb. Forty two femoral venous catheters (FVCs) were inserted into 40 infants and remained in situ for a median of seven days (range 1-29). Twenty seven (64\%) FVCs remained in place until no longer required, and eight (19\%) catheters were removed because of catheter related bloodstream infection.

Conclusions-FACs and FVCs are useful routes of vascular access in neonates when other sites are unavailable. Complications from femoral vessel catheterisation include transient lower limb ischaemia with FACs and catheter related bloodstream infection.

(Arch Dis Child Fetal Neonatal Ed 2001;85:F119-F122)
\end{abstract}

Keywords: femoral venous catheter; femoral arterial catheter; central venous access

Care Unit, Rosie

Hospital,

Addenbrooke's

Hospital, Cambridge, UK

A W R Kelsall

Neonatal Intensive

Care Unit, Liverpool

Women's Hospital

C W Yoxall

N V Subhedar

Correspondence to:

Dr Subhedar, Neonata

Intensive Care Unit,

Liverpool Women's Hospital,

Crown Street, Liverpool

L8 7SS, UK

nvsubhedar_wh@yahoo.com

Accepted 8 June 2001 peripheral intravenous catheters. ${ }^{3}$ When these routes have been exhausted, central venous catheters may be inserted surgically, but this often requires transfer to another hospital with specialist neonatal surgical and anaesthetic expertise. In an emergency, temporary vascular access can also be obtained by the intraosseus route. $^{4}$

Loss of venous access can be a significant problem when infants require long term intravenous treatment and nutrition. Failure to establish arterial access precludes continuous invasive monitoring of blood pressure and sampling of arterial blood gas, both of which are desirable in the management of ventilated neonates. ${ }^{5}$

The use of the femoral artery and vein for vascular access has traditionally been discouraged in neonates. Proximity to the groin is thought to increase the risk of infection. Intermittent puncture of the femoral vein may predispose to septic arthritis of the hip. ${ }^{6}$ The femoral artery is an end artery, and thromboembolic complications may produce distal ischaemia of the lower limb. Despite these theoretical concerns, the femoral vessels are often used during cardiac catheterisation, even in neonates, and the reported incidence of complications is low. ${ }^{7}$

Case series have been reported of femoral venous catheters inserted into neonates after surgical dissection ${ }^{89}$ or percutaneously through a needle..$^{11}$ To our knowledge, there are no reports on the use of modern catheters introduced into the femoral vessels using the Seldinger technique in neonates. We report our experience of the use of femoral arterial catheters (FACs) and femoral venous catheters (FVCs) during neonatal intensive care when alternative routes of access were unavailable.

\section{Methods}

Infants in whom an FAC or FVC was inserted were identified from two regional neonatal intensive care unit databases. Femoral catheters were inserted at the discretion of the clinician. Information was collected on the following: gestational age, birth weight, age and weight at insertion, reasons for insertion, duration in situ, complications, and indication for removal. Catheter related bloodstream infection was diagnosed when catheter and peripheral blood cultures were both positive for the same organism. Such infection was managed by either treatment with appropriate antibiotics through the catheter or its removal.

All the catheters were inserted by one of four trained operators who were experienced in the insertion technique. All FVCs and most FACs were inserted aseptically using the Seldinger 
Table 1 Patient details

\begin{tabular}{lll}
\hline & $\begin{array}{l}\text { Femoral venous catheters } \\
(n=42)\end{array}$ & $\begin{array}{l}\text { Femoral arterial catheters } \\
(n=23)\end{array}$ \\
\hline Gestational age (weeks) & $32(23-40)$ & $27(23-37)$ \\
Birth weight (g) & $1713(474-3710)$ & $845(550-3480)$ \\
Age at insertion (days) & $24(1-140)$ & $3(1-112)$ \\
Weight at insertion (g) & $2265(452-6600)$ & $1002(550-6600)$ \\
Duration in situ (days) & $7(1-29)$ & $3(1-8)$ \\
\hline
\end{tabular}

Each variable is expressed as median (range).

technique described below. ${ }^{12}$ In a small number of infants, femoral artery catheterisation was performed using a $24 \mathrm{G}$ cannula alone without another catheter being introduced over a guidewire. All infants had platelet counts above $50 \times 10^{9} / 1$, with normal coagulation studies. Ventilated infants were sedated with morphine sulphate $(100 \mu \mathrm{g} / \mathrm{kg}$ intravenously) sometimes in combination with a muscle relaxant. Nonventilated infants were sedated with either chloral hydrate $(50 \mathrm{mg} / \mathrm{kg}$ orally or rectally) or ketamine (2 $\mathrm{mg} / \mathrm{kg}$ intramuscularly). Local anaesthetic use depended on the operator. The inguinal region was cleaned using aqueous chlorhexidine and a sterile field established. The femoral artery was identified below the inguinal ligament as the key landmark. Several different types of central venous catheters are available and come prepackaged with all the equipment necessary for insertion. The following types of catheter were used: $20 \mathrm{G} 8 \mathrm{~cm}$ Leadercath and 22G $6 \mathrm{~cm}$ Leaderflex (Vygon, Aachen, Germany); 24G Jelco Cannulae (Johnson \& Johnson, Arlington, USA); Careflow 18G $10 \mathrm{~cm}$ (Becton Dickenson, Franklin Lakes, USA). The catheter length and number of lumens was chosen to suit the clinical circumstance.

SELDINGER TECHNIQUE

The insertion technique and anatomical landmarks have been described previously. ${ }^{10}$ The femoral vessels were initially catheterised from just below the inguinal ligament advancing

Table 2 Details of the cases in which femoral arterial catheters were used

\begin{tabular}{|c|c|c|c|c|c|}
\hline Subject & $\begin{array}{l}\text { Postnatal } \\
\text { age (days) }\end{array}$ & $\begin{array}{l}\text { Weight } \\
(g)\end{array}$ & $\begin{array}{l}\text { Gestational } \\
\text { age (weeks) }\end{array}$ & $\begin{array}{l}\text { Length of time } \\
\text { catheter in } \\
\text { place (days) }\end{array}$ & Reason for removal and complications \\
\hline 1 & 23 & 550 & 23 & 2 & Blocked, died later \\
\hline 2 & 12 & 576 & 24 & 3 & No longer required, died later \\
\hline 3 & 10 & 635 & 25 & 3 & Blocked, died later \\
\hline 4 & 2 & 642 & 25 & 2 & $\begin{array}{l}\text { Limb ischaemia, resolved when } \\
\text { catheter removed }\end{array}$ \\
\hline 5 & 4 & 657 & 26 & 3 & Died \\
\hline 6 & 2 & 680 & 26 & 7 & $\begin{array}{l}\text { Limb ischaemia, resolved when } \\
\text { catheter removed }\end{array}$ \\
\hline 7 & 2 & 758 & 29 & 7 & No longer required \\
\hline 8 & 1 & 758 & 29 & 6 & No longer required, died later \\
\hline 9 & 30 & 778 & 24 & 2 & Died \\
\hline 10 & 3 & 845 & 28 & 4 & No longer required \\
\hline 11 & 1 & 990 & 27 & 1 & Blocked, died later \\
\hline 12 & 3 & 990 & 27 & 1 & Died \\
\hline 13 & 23 & 1013 & 24 & 1 & $\begin{array}{l}\text { Limb ischaemia, resolved when } \\
\text { catheter removed }\end{array}$ \\
\hline 14 & 84 & 1569 & 26 & 4 & Blocked, died later \\
\hline 15 & 3 & 2000 & 34 & 5 & Blocked, died later \\
\hline 16 & 1 & 2330 & 37 & 2 & No longer required \\
\hline 17 & 19 & 2492 & 35 & 2 & Blocked \\
\hline 18 & 1 & 2590 & 35 & 2 & No longer required \\
\hline 19 & 7 & 2656 & 35 & 1 & $\begin{array}{l}\text { Limb ischaemia, resolved when } \\
\text { catheter removed }\end{array}$ \\
\hline 20 & 73 & 2870 & 27 & 8 & No longer required \\
\hline 21 & 3 & 3168 & 37 & 6 & No longer required \\
\hline 22 & 2 & 3370 & 35 & 2 & Fell out \\
\hline 23 & 112 & 6600 & 33 & 7 & Died \\
\hline
\end{tabular}

either a $20 \mathrm{G}$ introducer needle or a standard $22 \mathrm{G}$ intravenous cannula through the skin at an angle of $30-45^{\circ}$ in the direction of the umbilicus. The initial point of entry was either just above the femoral arterial pulse (for FACs) or just medial to it (for FVCs). Correct placement of the introducer needle or catheter was confirmed by the aspiration or free flow of blood. A guide wire was then introduced into the vessel and the needle or catheter removed with care to avoid dislodging the wire. The catheter was threaded over the wire and the guidewire removed. Aspiration of blood through the catheter confirmed successful insertion.

Catheters were sutured and/or secured in position using adhesive strips and covered with a transparent dressing. The site of insertion and the lower limb were inspected regularly to identify complications such as bleeding, venous engorgement, or ischaemia. When limb ischaemia was identified, catheters were removed within a few minutes. All catheters were connected to infusion pumps with Luer locked infusion tubing, and heparinised fluid was used for all infusions. In multilumen catheters, ports not being used were regularly flushed with heparinised saline to maintain patency.

\section{Results}

A total of 23 FACs and 42 FVCs were placed in 53 infants. Table 1 shows details of the patients, and tables 2 and 3 give details about the FACs and FVCs inserted respectively. Four infants had FACs and FVCs in situ simultaneously, and several infants had FACs and/or FVCs inserted on two or more separate occasions. Seventeen (30\%) of the infants died, but none of these deaths was caused by the femoral vessel catheterisation.

Femoral arterial catheterisation was associated with transient ischaemia of the distal limb in four cases (17\% (95\% confidence interval 5 to $38 \%$ )), but in all cases perfusion was rapidly restored once the catheter was promptly removed. There were no cases of gangrene $(0 \%$ ( $95 \%$ confidence intervals 0 to $15 \%)$ ). No cases of catheter related bloodstream infection were associated with FAC insertion. Six (26\%) arterial catheters were removed because of blockage. One catheter was accidentally dislodged without significant haemorrhage. The remaining $12(52 \%)$ catheters were removed when no longer required.

Femoral venous catheterisation was associated with venous engorgement of the distal limb in six cases $(14 \%)$. The swelling resolved completely within a week of catheter removal. Nine catheters $(21 \%)$ were associated with Staphylococcus epidermidis positive blood cultures; eight of these catheters were removed. Nine catheters $(21 \%)$ were removed because of blockage, and only one venous catheter was accidentally dislodged. In five (12\%) cases, a new catheter was resited over a guidewire, prolonging access through the femoral vein. Twenty seven $(64 \%)$ of the venous catheters remained in place until they were no longer required. 
Table 3 Details of the cases in which femoral venous catheters were used

\begin{tabular}{|c|c|c|c|c|c|}
\hline Subject & $\begin{array}{l}\text { Postnatal } \\
\text { age (days) }\end{array}$ & $\begin{array}{l}\text { Weight } \\
(g)\end{array}$ & $\begin{array}{l}\text { Gestational } \\
\text { age (weeks) }\end{array}$ & $\begin{array}{l}\text { Length of time } \\
\text { catheter in } \\
\text { place (days) }\end{array}$ & Reason for removal and complications \\
\hline 1 & 13 & 452 & 24 & 1 & Blocked \\
\hline 2 & 20 & 550 & 23 & 2 & No longer required, died later \\
\hline 3 & 2 & 576 & 24 & 8 & Line related sepsis, died later \\
\hline 4 & 12 & 576 & 24 & 3 & No longer required, died later \\
\hline 5 & 3 & 600 & 24 & 7 & Line related sepsis, died later \\
\hline 6 & 13 & 608 & 25 & 1 & Blocked \\
\hline 7 & 22 & 650 & 24 & 13 & Line related sepsis \\
\hline 8 & 75 & 758 & 29 & 15 & No longer required, died later \\
\hline 9 & 46 & 825 & 29 & 29 & Venous engorgement, sepsis \\
\hline 10 & 30 & 1022 & 26 & 5 & Blocked \\
\hline 11 & 84 & 1569 & 26 & 9 & Sepsis, died later \\
\hline 12 & 2 & 1596 & 33 & 5 & Died \\
\hline 13 & 86 & 1553 & 25 & 6 & Transient venous engorgement \\
\hline 14 & 40 & 1699 & 28 & 10 & No longer required \\
\hline 15 & 75 & 1761 & 26 & 5 & Blocked, venous engorgement, sepsis \\
\hline 16 & 58 & 1800 & 28 & 4 & No longer required \\
\hline 17 & 75 & 1829 & 29 & 15 & No longer required, died later \\
\hline 18 & 62 & 1850 & 28 & 7 & No longer required \\
\hline 19 & 8 & 2000 & 32 & 17 & Transient venous engorgement \\
\hline 20 & 7 & 2190 & 32 & 3 & Fell out, died later \\
\hline 21 & 6 & 2200 & 37 & 12 & No longer required \\
\hline 22 & 49 & 2200 & 32 & 13 & No longer required \\
\hline 23 & 1 & 2330 & 37 & 2 & No longer required \\
\hline 24 & 22 & 2368 & 34 & 6 & Line related sepsis \\
\hline 25 & 22 & 2560 & 33 & 15 & Transient venous engorgement \\
\hline 26 & 8 & 2656 & 35 & 9 & No longer required \\
\hline 27 & 3 & 2800 & 36 & 3 & Fell out \\
\hline 28 & 33 & 2800 & 37 & 4 & No longer required \\
\hline 29 & 50 & 3100 & 33 & 18 & Transient venous engorgement \\
\hline 30 & 8 & 3156 & 38 & 4 & Blocked \\
\hline 31 & 3 & 3168 & 37 & 6 & No longer required \\
\hline 32 & 3 & 3202 & 37 & 4 & No longer required \\
\hline 33 & 131 & 3351 & 31 & 10 & Infection, died later \\
\hline 34 & 140 & 3400 & 27 & 5 & No longer required \\
\hline 35 & 2 & 3400 & 37 & 5 & Fell out \\
\hline 36 & 124 & 3600 & 26 & 8 & No longer required \\
\hline 37 & 25 & 3750 & 39 & 9 & No longer required \\
\hline 38 & 29 & 3840 & 39 & 5 & No longer required \\
\hline 39 & 2 & 3850 & 40 & 12 & Line related sepsis, died later \\
\hline 40 & 100 & 3870 & 26 & 12 & $\begin{array}{l}\text { Local erythema, removed when no } \\
\text { longer required }\end{array}$ \\
\hline 41 & 32 & 4300 & 39 & 26 & No longer required \\
\hline 42 & 112 & 6600 & 33 & 7 & Died \\
\hline
\end{tabular}

\section{Discussion}

We here report our experience of the use of FACs and FVCs in two regional neonatal intensive care units. There were no major complications from the use of these catheters, and minor complications were relatively rare and in all cases resolved rapidly.

Even though there were no major catheter related complications in this study, we cannot rule out the possibility of serious and therefore clinically important complications occurring occasionally. Gangrene and loss of the lower limb have been reported previously with the use of FACs in children, and the incidence of this complication in very low birthweight infants is likely to be higher. Femoral catheters have not been used widely in extremely low birthweight infants in the past because of the expected risks. We did not show a high incidence of adverse effects, but catheterisation of infants weighing less than $1000 \mathrm{~g}$ did not last for long periods, often because infants died for reasons unrelated to their vascular catheters. The inevitable high mortality in this group means that the incidence of medium and long term complications cannot be determined, and follow up of these infants is therefore important.

In general, FACs were inserted early in the course of respiratory illness (median age three days), when invasive blood pressure monitoring and/or arterial blood sampling were deemed essential for clinical management. In contrast, FVCs tended to be inserted later (median age 24 days), to provide secure long term venous access when other routes were no longer available. They remained in place for longer than FACs (median of seven days versus three days), presumably because intra-arterial catheters were removed once an infant's cardiorespiratory status had stabilised.

In our series, although the complication rate with FVCs was relatively high, the complications were usually minor. Transient limb engorgement and catheter related bloodstream infection were the most common problems. This is in keeping with other studies reporting complications with central venous catheters in neonates ${ }^{132}$ and with other reports of the use of percutaneous FVCs using a different insertion technique. $^{1011}$ The complication rate with FACs was similar to that with FVCs, with transient distal ischaemia and catheter blockage occurring most commonly. All the complications described above were reversible and responded to removal of the catheter or appropriate treatment. Overall, in difficult circumstances in which no alternative vascular access was readily available, over half of all FVCs and FACs remained in situ until they were no longer required. We acknowledge that one limitation of our study is the lack of a control group of infants in whom alternative vascular access was used. By definition, our study population was one in which no such vascular routes were available, and therefore there could be no directly comparable control group. Instead we chose to compare our complication rates with those previously described in the literature.

All cases of catheter related bloodstream infection occurred in infants with FVCs. This is probably because FVCs were used for older infants than were FACs and remained in situ for longer, thus increasing the risk of infection. Infants with catheter related bloodstream infection responded to appropriate antibiotic treatment and/or catheter removal. No cases of septic arthritis of the hip were identified in this series.

There are several case reports describing the use of FVCs in neonates. Hogan and Pulito ${ }^{8}$ described the surgical cut down insertion of Broviac catheters through the saphenous and femoral veins under local anaesthetic in 84 infants, and Meland et $a l^{9}$ reported the use of a similar technique in a series of 35 neonates. In these series, complications occurred in about $50 \%$ of cases, but $62-83 \%$ of catheters remained in situ until no longer required. There are two reports of percutaneous femoral venous catheter insertion using a modified Seldinger technique with a fine catheter threaded through an introducer needle. In the first study, silastic catheters were inserted into 44 infants $;{ }^{10}$ in the other, silicone rubber catheters were inserted into 63 infants. ${ }^{11}$ The incidence of complications in both studies was small, with most catheters being removed electively. Our report is different from these studies in a number of ways. Firstly, all FVCs and most FACs were inserted using the standard 
Seldinger technique. We have also described femoral catheterisation in a much smaller and more immature group of infants than that previously reported. Furthermore, in our series, the femoral route was only used as a last resort in sick babies when no alternative vascular access was available.

This study illustrates that FACs and FVCs can be used to obtain vascular access and permit continuous invasive monitoring in sick neonates when other routes have failed or been exhausted. As no major complications occurred in this series, we conclude that the risk of serious adverse events with this technique is low in experienced hands. This study provides support for the use of femoral vessel catheterisation in newborn infants. We would recommend that insertion of femoral catheters in the neonate should only be performed by trained experienced personnel.

1 Brain AJ, Roberton NRJ, Rennie JM. Iatrogenic disorders. In: Rennie JM, Roberton NRC, eds. Textbook of neonatolog. 3rd ed. Edinburgh: Churchill-Livingstone, 1999:917-38.

2 Neubauer AP. Percutaneous intravenous access in the neonate: experience with 535 silastic catheters. Acta Paediatr 1995;84:756-60.
3 Narendran V, Gupta G, Todd DA, et al. Bacterial colonisation of indwelling vascular catheters in newborn infants. $\mathcal{F}$ Paediatr Child Health 1996;32:391-6.

4 Kelsall AWR. Resuscitation with intraosseus lines in neonatal units. Arch Dis Child 1993;68:324-5.

5 Report of a joint working group of the British Association of Perinatal Medicine and the Research Unit of the Royal College of Physicians. Development of audit measures and College of Physicians. Development of audit measures and
guidelines for good practice in the management of neonaguidelines for good practice in the management of neona-
tal respiratory distress syndrome. Arch Dis Child tal respiratory

6 Asnes RS, Arender GM. Septic arthritis of the hip: a complication of femoral venipuncture. Pediatrics 1966;38:837-41.

7 Rosenthal A, Anderson M, Thomson SJ, et al. Superficial femoral artery cannulation. American fournal of Disease of Children 1972;124:240-2.

8 Hogan L, Pulito AR. Broviac central venous catheters inserted via the saphenous or femoral vein in the NICU under local anesthesia. F Pediatr Surg 1992;27:1185-8.

9 Meland NB, Wilson W, Soontharotoke CY, et al. Saphenofemoral venous cutdowns in the premature infant. f Pediatr Surg 1986;21:341-3.

10 Serrao PR, Jean-Louis J, Godoy J, et al. Inferior vena cava catheterization in the neonate by the percutaneous femoral vein method. F Perinatol 1996;16:129-32.

11 Abdulla F, Dietrich KA, Pramanik AK. Percutaneous femoral venous catheterization in preterm neonates. $\mathcal{F}$ Pediatr 1990;117:788-91.

12 Conahan TJ, Schwartz AJ, Greer RT. Percutaneous catheter introduction: the Seldinger technique. $\mathscr{f} A M A$ 1977;237:445-7.

13 Pandit PB, Pandit FA, Govan J, et al. Complications associated with surgically placed central venous catheters in low birth weight neonates. F Perinatol 1999;19:106-9. 